\title{
Implementing Two-Party Computation Efficiently with Security Against Malicious Adversaries ${ }^{\star}$
}

\author{
Yehuda Lindell ${ }^{1}$, Benny Pinkas ${ }^{2}$, and Nigel P. Smart ${ }^{3}$ \\ 1 Dept. Of Computer Science, \\ Bar Ilan University, \\ Ramat Gan, Israel, \\ lindell@cs.biu.ac.il \\ 2 Dept. of Computer Science, \\ University of Haifa \\ Haifa 31905, Israel \\ benny@pinkas . net \\ 3 Dept. Computer Science, \\ University of Bristol, \\ Woodland Road, Bristol, BS8 1UB, United Kingdom, \\ nigel@cs.bris.ac.uk
}

\begin{abstract}
We present an implementation of the protocol of Lindell and Pinkas for secure two-party computation which is secure against malicious adversaries [13]. This is the first running system which provides security against malicious adversaries according to rigorous security definition and without using the random oracle model. We ran experiments showing that the protocol is practical. In addition we show that there is little benefit in replacing subcomponents secure in the standard model with those which are only secure in the random oracle model. Throughout we pay particular attention to using the most efficient subcomponents in the protocol, and we select parameters for the encryption schemes, commitments and oblivious transfers which are consistent with a security level equivalent to AES-128.
\end{abstract}

\section{Introduction}

Secure multi-party computation is a process which allows multiple participants to implement a joint computation that, in real life, may only be implemented using a trusted party. The participants, each with its own private input, communicate without the help of any trusted party, and can compute any function without revealing information about the inputs (except for the value of the function). A

\footnotetext{
* The first author was supported by The Israel Science Foundation (grant No. 781/07) and by an Infrastructures grant from the Israeli Ministry of Science. The other authors were supported by the European Union under the FP7-STREP project CACE. The second author was also supported by The Israel Science Foundation (grant No. 860/06).
} 
classic example of such a computation is the Millionaires' problem, in which two millionaires want to know who is richer, without revealing their actual worth.

Multi-party computation has been considered by the theoretical cryptography community for a long time, starting with the pioneering work of Yao [24] in 1986. Yao's garbled circuit construction is relatively simple, and runs in a constant number of rounds. Yao's construction still remains the most attractive choice for generic secure two-party computation.

In recent years attention has focused on whether the theoretical work has any practical significance. In the two-party case the main contribution has been the FairPlay compiler [15], which is a generic tool translating functions written in a special high-level language to Java programs which execute a secure protocol implementing them. There are two major drawbacks with the current FairPlay implementation. Firstly it only provides weak security against malicious adversaries (where reducing the cheating probability to $1 / k$ requires increasing the overhead by a factor of $k$ ), and has no proof of security (in particular, it is clear that it cannot be proven secure under simulation-based definitions). As such, its usage can only be fully justified for providing security against honest but curious (aka semi-honest) adversaries. ${ }^{1}$ Secondly it does not make use of the latest and most efficient constructions of its various component parts.

In recent years the theoretical community has considered a number of ways of providing a variant of Yao's protocol which is secure against malicious adversaries. In the current paper we examine one of the more recent and efficient protocols for providing security for Yao's protocol against malicious adversaries, namely the protocol of Lindell and Pinkas [13] which is proved to be secure according to a standard simulation based definition, and as such can be securely used as a primitive in more complex protocols (see [8, Chapter 7], which in turn follows [6]).

Our work presents the following contributions:

- We provide an efficient implementation of the protocol of [13], which is secure against malicious adversaries. This is, to our best knowledge, the first implementation of a generic two-party protocol that is secure against malicious adversaries according to a standard simulation based definition. The implementation demonstrates the feasibility of the use of such protocols.

- We derive a number of optimizations and extensions to the protocol and to the different primitives that it uses. Unlike prior implementations we pay particular attention to using the most efficient constructions for the various components. For example we use elliptic curve based oblivious transfer protocols instead of finite field discrete logarithm based protocols.

\footnotetext{
${ }^{1}$ The cryptographic community denotes adversaries which can operate arbitrarily as "malicious". Semi-honest (or honest but curious) adversaries are supposed to follow the protocol that normal users are running, but they might try to gain information from the messages they receive in the protocol. It is, of course, easier to provide security against semi-honest adversaries.
} 
- We also examine the difference between using protocols which are secure in the random oracle model (ROM) and protocols in the standard model. ${ }^{2}$ Of particular interest is that our results show that there appears to be very little benefit in using schemes which are secure in the ROM as opposed to the standard model. ${ }^{3}$

\subsection{Related Work}

Research on security against malicious adversaries for computationally secure protocols started with the seminal GMW compiler [9]. As we have mentioned, we base our work on the protocol of [13], and we refer the reader to that work for a discussion of other approaches for providing security against malicious adversaries (e.g., $[14,11,23])$. We note that a simulation based proof of security (as in [13]) is essential in order to enable the use of a protocol as a building block in more complex protocols, while proving the security of the latter using general composition theorems like those of $[6,8]$. This is a major motivation for the work we present in this paper, which enables efficient construction of secure function evaluation primitives that can be used by other protocols. (For example, the secure protocol of [2] for finding the $k^{\text {th }}$ ranked element is based on invoking several secure computations of comparisons, and provides simulation based security against malicious adversaries if the invoked computations have a simulation based proof. Our work enables to efficiently implement that protocol.)

The first generic system implementing secure two-party computation was FairPlay [15], which provided security against semi-honest adversaries and limited security against malicious adversaries (see discussion above). FairPlayMP is a generic system for secure multi-party computation, which only provides security against semi-honest adversaries [3]. Another system in the multi-party scenario is SIMAP, developing a secure evaluation of an auction using general techniques for secure computation $[5,4]$. It, too, supports only security against semi-honest adversaries.

\subsection{Paper Structure}

Section 2 introduces Yao's protocol for secure two-party computation, while Section 3 presents the protocol of [13] which is secure against malicious adversaries.

\footnotetext{
${ }^{2}$ A random oracle is a function which is modeled as providing truly random answers. This abstraction is very useful for proving the security of cryptographic primitives. However, given any specific implementation of a function (known to the users who use it), this assumption no longer holds. Therefore it is preferable to prove security in the standard model, namely without using any random oracle.

${ }^{3}$ This is surprising since for more traditional cryptographic constructions, such as encryption schemes or signature schemes, the random oracle constructions are almost always twice as efficient in practice compared to the most efficient standard model schemes known. Part of the reason for the extreme efficiency of our standard model constructions is our use of a highly efficient oblivious transfer protocol which reduces the amortized number of zero-knowledge proofs which are required to be performed.
} 
Section 4 presents the different efficient sub-protocols that we used. Finally, Section 5 presents the results of our experiments.

\section{Yao's Garbled Circuit}

Two-party secure function evaluation makes use of the famous garbled circuit construction of Yao [24]. In this section we briefly overview the idea. Note, however, that the following basic protocol is not secure against malicious adversaries, which is why the advanced protocol in the next section is to be preferred. The basic idea is to encode the function to be computed via a Binary circuit and then to securely evaluate the circuit on the players' inputs.

We consider two parties, denoted as $P_{1}$ and $P_{2}$, who wish to compute a function securely. Suppose we have a simple Binary circuit consisting of a single gate, the extension to many gates given what follows is immediate. The gate has two input wires, denoted $w_{1}$ and $w_{2}$, and an output wire $w_{3}$. Assume that $P_{1}$ knows the input to wire $w_{1}$, which is denoted $b_{1}$, and that $P_{2}$ knows the input to wire $w_{2}$, which is denoted $b_{2}$. We assume that each gate has a unique identifier Gid (this is to enable circuit fan out of greater than one, i.e. to enable for the output wire of a gate to be used in more than one other gate). We want $P_{2}$ to determine the value of the gate on the two inputs without $P_{1}$ learning anything, and without $P_{2}$ determining the input of $P_{1}$ (bar what it can determine from the output of the gate and its own input). We suppose that the output of the gate is given by the function $G\left(b_{1}, b_{2}\right) \in\{0,1\}$

Yao's construction works as follows. $P_{1}$ encodes, or garbles, each wire $w_{i}$ by selecting two different cryptographic keys $k_{i}^{0}$ and $k_{i}^{1}$ of length $t$, where $t$ is a computational security parameter which suffices for the length of a symmetric encryption scheme. In addition to each wire it associates a random permutation $\pi_{i}$ of $\{0,1\}$. The garbled value of the wire $w_{i}$ is then represented by the pair $\left(k_{i}^{b_{i}}, c_{i}\right)$, where $c_{i}=\pi_{i}\left(b_{i}\right)$.

An encryption function $E_{k_{1}, k_{2}}^{s}(m)$ is selected which has as input two keys of length $t$, a message $m$, and some additional information $s$. The additional information $s$ must be unique per invocation of the encryption function (i.e., used only once for any choice of keys). The precise encryption functions used are described in Section 4.1. The gate itself is then replaced by a four entry table indexed by the values of $c_{1}$ and $c_{2}$, and given by

$$
c_{1}, c_{2}: E_{k_{1}^{b_{1}}, k_{2}^{b_{2}}}^{\mathrm{Gid}\left\|c_{2}\right\| c_{2}}\left(k_{3}^{G\left(b_{1}, b_{2}\right)} \| c_{3}\right),
$$

where $b_{1}=\pi_{1}^{-1}\left(c_{1}\right), b_{2}=\pi_{2}^{-1}\left(c_{2}\right)$, and $c_{3}=\pi_{3}\left(G\left(b_{1}, b_{2}\right)\right)$. Note that each entry in the table corresponds to a combination of the values of the input wires, and contains the encryption of the garbled value corresponding to these values of the input wires, and the corresponding $c$ value. The resulting look up table (or set of look up tables in general) is called the Garbled Circuit.

$P_{1}$ then sends to $P_{2}$ the garbled circuit, its input value $k_{1}^{b_{1}}$, the value $c_{1}=$ $\pi_{1}\left(b_{1}\right)$, and the mapping from the set $\left\{k_{3}^{0}, k_{3}^{1}\right\}$ to $\{0,1\}$ (i.e. the permutation 
$\left.\pi_{3}\right) . P_{1}$ and $P_{2}$ engage in an oblivious transfer (OT) protocol so that $P_{2}$ learns the value of $k_{2}^{b_{2}}, c_{2}$ where $c_{2}=\pi_{2}\left(b_{2}\right) . P_{2}$ can then decrypt the entry in the look up table indexed by $\left(c_{1}, c_{2}\right)$ using $k_{1}^{b_{1}}$ and $k_{2}^{b_{2}}$; this will reveal the value of $k_{3}^{G\left(b_{1}, b_{2}\right)} \| c_{3}$ and $P_{2}$ can determine the value of $G\left(b_{1}, b_{2}\right)$ by using the mapping $\pi_{3}^{-1}$ from the set $c_{3}$ to $\{0,1\}$.

In the general case the circuit consists of multiple gates. $P_{1}$ chooses random garbled values for all wires and uses them for constructing tables for all gates. It sends these tables (i.e., the garbled circuit) to $P_{2}$, and in addition provides $P_{2}$ with the garbled values and the $c$ values of $P_{1}$ 's inputs, and with the permutations $\pi$ used to encode the output wires of the circuit. $P_{2}$ uses invocations of oblivious transfer to learn the garbled values and $c$ values of its own inputs to the circuits. Given these values $P_{2}$ can evaluate the gates in the first level of the circuit, and compute the garbled values and the $c$ values of the values of their output wires. It can then continue with this process and compute the garbled values of all wires in the circuit. Finally, it uses the $\pi$ permutations of the output wires of the circuit to compute the real output values of the circuit.

Traditionally, for example in hardware design, one uses circuits which are constructed of simple gates which take at most two inputs and produce as most one output. In a Yao circuit a gate which takes $n$ inputs and produces $m$ outputs is encoded as a look up table which has $2^{n}$ rows, each consisting of a string of $O(m \cdot t)$ bits (where $t$ is the security parameter which denotes the length of a key). Hence, it is often more efficient to use non-standard gates in a Yao circuit construction. For example a traditional circuit component consisting of $k 2$-to-1 gates, with $n$ input and $m$ output wires can be more efficiently encoded as a single $n$-to- $m$ gate if $4 k>2^{n}$. In what follows we therefore assume the more suitable $n$-to- $m$ gate construction. The extension of the above gate description to this more general case is immediate.

\section{The Lindell-Pinkas Protocol}

The protocol was presented in [13] and was proved there to be secure according to the real/ideal-model simulation paradigm $[6,8]$. The proof is in the standard model, with no random oracle model or common random string assumptions. We describe below the protocol in some detail, for full details see [13]. We remark that this description is not essential in order to understand the results of our paper. The important things to note are the basic structure of the protocol, as described in the next paragraph, and the fact that the protocol is based on the use of different types of commitments (statistically binding, statistically hiding, and computational), and of an oblivious transfer protocol. We describe the implementation of these primitives in Section 4.

The basic structure of the protocol: The protocol proceeds in the following steps. It has statistical security parameters $s_{1}$ and $s_{2}$. We replace $P_{2}$ 's input wires with a new set of $O\left(s_{2}\right)$ input wires, and change the original circuit by adding to it a new part which translates the values of the new input wires to those of 
the original wires. Then $P_{1}$ generates $s_{1}$ copies of Yao circuits and passes them to $P_{2}$, along with $O\left(s_{1}^{2}\right)$ commitments to the inputs. The input decommitments for $P_{1}$ 's inputs are transferred to $P_{2}$ via a batched oblivious transfer. Finally, after executing a number of cut-and-choose checks on the transferred circuits and commitments, $P_{2}$ evaluates half of the circuits and determines the output value as the majority value of the outputs of these circuits. One of the contributions of this paper is to examine each of the above operations in turn and optimize the parameters and components used in the Lindell-Pinkas description.

\subsection{The Protocol in Detail}

As explained in [13] it suffices to present a protocol for the case where the output is learnt by $P_{2}$ and $P_{1}$ learns nothing. We consider the computation of $f(x, y)$ where $P_{1}$ 's input is $x \in\{0,1\}^{n}$ and $P_{2}$ 's input is $y \in\{0,1\}^{n}$.

The protocol is parameterized by two statistical security parameters $s_{1}$ and $s_{2}$. (In [13] these are a single statistical security parameter but we shall see later that in order to optimize performance these parameters really need to be treated separately.) The protocol takes as input a circuit description $C^{0}(x, y)$ which describes the function $f(x, y)$. We use the notation $\operatorname{com}_{b}$ to refer to a statistically binding commitment scheme, $\mathrm{com}_{h}$ to refer to a statistically hiding commitment scheme, and $\operatorname{com}_{c}$ to refer to a commitment scheme which is only computationally binding and hiding. See Section 4 for our precise choice of these protocols.

The protocol itself is quite elaborate, but, as demonstrated in Section 5, it can be implemented quite efficiently.

0. Circuit construction: The parties replace $C^{0}$, in which $P_{2}$ has $n$ input wires, with a circuit $C$ in which $P_{2}$ has $\ell$ input wires, where $\ell=\max \left(4 n, 8 s_{2}\right)$. The only difference between the circuits is that each original input wire of $P_{2}$ in $C^{0}$ is replaced with an internal value which is computed as the exclusiveor of a random subset of the $\ell$ input wires of $C$. (Given an input to the original circuit, $P_{2}$ should therefore choose a random input to the new circuit, subject to the constraint that the internal values are equal to the original input values.) The exact construction is presented in Section 5.2 of [13]. (In order to avoid unnecessary extra gates in the circuit segment that computes the original input wires as a function of the new input wires, we designed the exact wiring using a variant of structured Gaussian elimination.)

We let the new input wires of $P_{2}$ be given by $\hat{y} \leftarrow \hat{y}_{1}, \ldots, \hat{y}_{\ell}$

1. Commitment construction: $P_{1}$ constructs the circuits and commits to them, as follows: ${ }^{4}$

(a) $P_{1}$ constructs $s_{1}$ independent copies of a garbled circuit of $C$, denoted $G C_{1}, \ldots, G C_{s_{1}}$. ${ }^{4}$ In [13] this commitment is done with a perfectly binding commitment scheme, how-
ever one which is computationally binding will suffice to guarantee security. 
(b) $P_{1}$ commits to the garbled values of the wires corresponding to $P_{2}$ 's input to each circuit. That is, for every input wire $i$ corresponding to an input bit of $P_{2}$, and for every circuit $G C_{r}, P_{1}$ computes the ordered pair

$$
\left(c_{i, r}^{0}, c_{i, r}^{1}\right) \leftarrow\left(\operatorname{com}_{c}\left(k_{i, r}^{0}\right), \operatorname{com}_{c}\left(k_{i, r}^{1}\right)\right),
$$

where $k_{i, r}^{b}$ is the garbled value associated with $b$ on input wire $i$ in circuit $G C_{r}$. We let $\left(d c_{i, r}^{0}, d c_{i, r}^{1}\right)$ denote the associated decommitment values.

(c) $P_{1}$ computes commitment-sets for the garbled values that correspond to its own inputs to the circuits. That is, for every wire $i$ that corresponds to an input bit of $P_{1}$, it generates $s_{1}$ pairs of commitment sets $\left\{W_{i, j}, W_{i, j}^{\prime}\right\}_{j=1}^{s_{1}}$, in the following way:

Denote by $k_{i, r}^{b}$ the garbled value that was assigned by $P_{1}$ to the value $b \in\{0,1\}$ of wire $i$ in $G C_{r}$. Then, $P_{1}$ chooses $b \leftarrow\{0,1\}$ and computes

$$
\begin{aligned}
& W_{i, j} \leftarrow\left\langle\operatorname{com}_{c}(b), \operatorname{com}_{c}\left(k_{i, 1}^{b}\right), \ldots, \operatorname{com}_{c}\left(k_{i, s_{1}}^{b}\right)\right\rangle, \\
& W_{i, j}^{\prime} \leftarrow\left\langle\operatorname{com}_{c}(1-b), \operatorname{com}_{c}\left(k_{i, 1}^{1-b}\right), \ldots, \operatorname{com}_{c}\left(k_{i, s_{1}}^{1-b}\right)\right\rangle .
\end{aligned}
$$

There are a total of $n \cdot s_{1}$ commitment-sets ( $s_{1}$ per input wire). We divide them into $s_{1}$ supersets, where superset $S_{j}$ is defined to be the set containing the $j$ th commitment set for all wires. Namely, it is defined as $S_{j}=\left\{\left(W_{k, j}, W_{k, j}^{\prime}\right)\right\}_{k=1}^{n}$.

2. Oblivious transfers: For every input bit of $P_{2}$, parties $P_{1}$ and $P_{2}$ run a 1-out-of-2 oblivious transfer protocol in which $P_{2}$ receives the garbled values for the wires that correspond to its input bit (in every circuit).

Let $i_{1}, \ldots, i_{w}$ be the input wires that correspond to $P_{2}$ 's input, then, for every $j=1, \ldots, w$, parties $P_{1}$ and $P_{2}$ run a 1-out-of-2 OT protocol in which:

(a) $P_{1}$ 's input is the pair of vectors $\left[d c_{i_{j}, 1}^{0}, \ldots, d c_{i_{j}, s_{1}}^{0}\right]$, and $\left[d c_{i_{j}, 1}^{1}, \ldots, d c_{i_{j}, s_{1}}^{1}\right]$.

(b) $P_{2}$ 's input are the bits $\hat{y}_{j}$, and its output should be $\left[d c_{i_{j}, 1}^{\hat{y}_{j}}, \ldots, d c_{i_{j}, s_{1}}^{\hat{y}_{j}}\right]$.

3. Send Circuits And Commitments: $P_{1}$ sends to $P_{2}$ the garbled circuits, as well as all of the commitments that it prepared above.

4. Prepare Challenge Strings: $:^{5}$

(a) $P_{2}$ chooses a random string $\rho_{2} \leftarrow\{0,1\}^{s_{1}}$ and sends $\operatorname{com}_{h}\left(\rho_{2}\right)$ to $P_{1}$.

(b) $P_{1}$ chooses a random string $\rho_{1} \in\{0,1\}^{s_{1}}$ and sends $\operatorname{com}_{b}\left(\rho_{1}\right)$ to $P_{2}$.

(c) $P_{2}$ decommits, revealing $\rho_{2}$.

(d) $P_{1}$ decommits, revealing $\rho_{1}$.

(e) $P_{1}$ and $P_{2}$ set $\rho \leftarrow \rho_{1} \oplus \rho_{2}$.

The above steps are run a second time, defining an additional string $\rho^{\prime}$.

5. Decommitment Phase for CheCK-CIRCUits: We refer to the circuits for which the corresponding bit in $\rho$ is 1 as check-circuits, and we refer to the other circuits as evaluation-circuits. Likewise, if the $j$ th bit of $\rho^{\prime}$ equals 1 , then all commitments sets in superset $S_{j}=\left\{\left(W_{i, j}, W_{i, j}^{\prime}\right)\right\}_{i=1}^{n}$ are referred to as check-sets; otherwise, they are referred to as evaluation-sets.

For every check-circuit $G C_{r}$, party $P_{1}$ operates in the following way:

\footnotetext{
${ }^{5}$ In [13] it is proposed to use perfectly binding and computationally hiding commitments here, but statistically binding and computationally hiding commitments actually suffice.
} 
(a) For every input wire $i$ corresponding to an input bit of $P_{2}$, party $P_{1}$ decommits to the pair $\left(c_{i, r}^{0}, c_{i, r}^{1}\right)$.

(b) For every input wire $i$ corresponding to an input bit of $P_{1}$, party $P_{1}$ decommits to the appropriate values in the check-sets $\left\{W_{i, j}, W_{i, j}^{\prime}\right\}$.

For every pair of check-sets $\left(W_{i, j}, W_{i, j}^{\prime}\right)$, party $P_{1}$ decommits to the first value in each set i.e., to the value that is supposed to be a commitment to the indicator bit, $\operatorname{com}(0)$ or $\operatorname{com}(1))$.

6. Decommitment phase for $P_{1}$ 's input in evaluation-circuits: $P_{1}$ decommits to the garbled values that correspond to its inputs in the evaluationcircuits.

7. Correctness and Consistency Checks: Player $P_{2}$ performs the following checks; if any of them fails it aborts.

(a) Checking correctness of the check-circuits: $P_{2}$ verifies that each checkcircuit $G C_{i}$ is a garbled version of $C$.

(b) Verifying $P_{2}$ 's input in the check-circuits: $P_{2}$ verifies that $P_{1}$ 's decommitments to the wires corresponding to $P_{2}$ 's input values in the checkcircuits are correct, and agree with the logical values of these wires (the indicator bits). $P_{2}$ also checks that the inputs it learned in the oblivious transfer stage for the check-circuits correspond to its actual input.

(c) Checking $P_{1}$ 's input to evaluation-circuits: Finally, $P_{2}$ verifies that for every input wire $i$ of $P_{1}$ the following two properties hold:

i. In every evaluation-set, $P_{1}$ chooses one of the two sets and decommitted to all the commitments in it which correspond to evaluationcircuits.

ii. For every evaluation-circuit, all of the commitments that $P_{1}$ opened in evaluation-sets commit to the same garbled value.

8. Circuit evaluation: If any of the above checks fails, $P_{2}$ aborts and outputs $\perp$. Otherwise, $P_{2}$ evaluates the evaluation circuits (in the same way as for the semi-honest protocol of Yao). It might be that in certain circuits the garbled values provided for $P_{1}$ 's inputs, or the garbled values learned by $P_{2}$ in the OT stage, do not match the tables and so decryption of the circuit fails. In this case $P_{2}$ also aborts and outputs $\perp$. Otherwise, $P_{2}$ takes the output that appears in most circuits, and outputs it.

\subsection{The Statistical Security Parameters}

The protocol uses two statistical security parameters, $s_{1}$ and $s_{2}$. The parameter $s_{1}$ is mainly used to prevent $P_{1}$ from changing the circuit that is evaluated, or providing inconsistent inputs to different copies of the circuit. The protocol requires $P_{1}$ to provide $s_{1}$ copies of the garbled circuit, and provide $\left(s_{1}\right)^{2}$ commitments for each of its input bits. The security proof in [13] shows that a corrupt $P_{1}$ can cheat with a success probability that is exponentially small in $s_{1}$. The original proof in [13] bounds the cheating probability at $2^{-s_{1} / 17}$, which would require a large value of $s_{1}$ in order to provide a meaningful security guarantee. We conjecture that a finer analysis can provide a bound of $2^{-s_{1} / 4}$, and in the full version of this paper we intend to prove this; this conjecture is based on an 
analysis of a similar problem that was shown in [10]. A bound of $2^{-s_{1} / 4}$ would mean that a relatively moderate value of $s_{1}$ can be used. ${ }^{6}$

The parameter $s_{2}$ is used to prevent a different attack by $P_{1}$, in which it provides corrupt values to certain inputs of the oblivious transfer protocol and then uses $P_{2}$ 's reaction to these values to deduce information about $P_{2}$ 's inputs (see [13] for details). It was shown that setting the number of new inputs to be $\ell=\max \left(4 n, 8 s_{2}\right)$ bounds the success probability of this type of attack by $2^{-s_{2}}$. The values of $s_{1}$ and $s_{2}$ should therefore be chosen subject to the constraint that the total success probability of a cheating attempt, $\max \left(2^{-s_{1} / 4}, 2^{-s_{2}}\right)$, is acceptable. Therefore, one should set $s_{1}=4 s_{2}$.

\subsection{Optimizing the Protocol Components}

The protocol uses many components, which affect its overall overhead. These include the encryption scheme, the commitment schemes, and oblivious transfer. Much of our work was concerned with optimizing these components, in order to improve the performance of the entire protocol. We describe in the next section the different optimizations that we applied to the different components.

\section{Subprotocols}

To implement the above protocol requires us to define a number of sub-protocols: various commitment schemes, OT protocols and encryption schemes. In what follows we select the most efficient schemes we know of, in both the random oracle model (ROM) and the standard model. We assume that the concrete computational security parameter (as opposed to the statistical security parameter) is given by $t$. By this we mean that we select primitives which have security equivalent to $t$ bits of block cipher security. Thus we first select an elliptic curve $E$ of prime order $q \approx 2^{2 t}$, and a symmetric cryptographic function with a $t$-bit key.

Elliptic curve. We let $\langle P\rangle=\langle Q\rangle=E$, an elliptic curve of prime order $q \approx 2^{2 t}$, where no party knows the discrete logarithm of $Q$ with respect to $P$.

Symmetric cryptographic function. The function that will be used for symmetric key cryptography is defined as a key derivation function $\operatorname{KDF}(m, l)$, which takes an input string $m$ and outputs a bit string of length $l$. We use the KDF defined in ANSI X9.63, which is the standard KDF to use in the elliptic curve community [19]. It is essentially implemented as encryption in CTR mode where the encryption function is replaced by the SHA-1 hash function.

\footnotetext{
${ }^{6}$ The experiments in Section 5 assume a bound of $2^{-s_{1} / 4}$. The overhead of different parts of the protocol is either linear or quadratic in $s_{1}$. If we end up using a worse bound of $2^{-s_{1} / c}$, where $4<c \leq 17$, the timings in the experiments will be increased by factor in the range $c / 4$ to $(c / 4)^{2}$.
} 


\subsection{Encryption Scheme for Garbled Circuits}

The encryption scheme $E_{k_{1}, k_{2}}^{s}(m)$ used to encrypt the values in the Yao circuit is defined by the algorithms in Figure 1 . We assume that $k_{i} \in\{0,1\}^{t}$. The ROM version is secure on the assumption that the function KDF is modelled as a random oracle, whilst the standard model scheme is secure on the assumption that $\operatorname{KDF}(k \| s, l)$ is a pseudo-random function, when considered as a function on $s$ keyed by the key $k$. We remark that the encryption is secure as long as the string $s$ is used only once for any choice of key $k$. Note that the non-ROM version requires two invocations of the $\mathrm{KDF}$, since we do not know how to analyze the security of a pseudo-random function if part of its key is known to an adversary (namely, if we use $\operatorname{KDF}\left(k_{1}\left\|k_{2}\right\| s,|m|\right)$, where $\mathrm{KDF}$ is modeled as a pseudo-random function, $k_{2}$ is secret and $k_{1}$ is known to an adversary, we cannot argue that the output is pseudo-random).

Figure 1 ROM and non-ROM encryption algorithms for the Yao circuits

Input: Keys $k_{1}, k_{2}$ of length $t$, and a string $s$. For encryption an $l$-bit message $m$ in also given. For decryption, an $l$-bit ciphertext $c$ is given.

\section{ROM Version}

$$
\text { Encryption } E_{k_{1}, k_{2}}^{s}(m)
$$

1. $k \leftarrow \operatorname{KDF}\left(k_{1}\left\|k_{2}\right\| s,|m|\right)$.

2. $c \leftarrow k \oplus m$.

\section{Decryption}

1. $k \leftarrow \operatorname{KDF}\left(k_{1}\left\|k_{2}\right\| s,|m|\right)$.

2. $m \leftarrow k \oplus c$

3. Return $m$.

\section{Non-ROM Version}

Encryption $E_{k_{1}, k_{2}}^{s}(m)$

1. $k \leftarrow \operatorname{KDF}\left(k_{1} \| s,|m|\right)$.

2. $k^{\prime} \leftarrow \operatorname{KDF}\left(k_{2} \| s,|m|\right)$.

3. $c \leftarrow m \oplus k \oplus k^{\prime}$

\section{Decryption}

1. $k \leftarrow \operatorname{KDF}\left(k_{1} \| s,|c|\right)$.

2. $k^{\prime} \leftarrow \operatorname{KDF}\left(k_{2} \| s,|c|\right)$.

3. $m \leftarrow c \oplus k \oplus k^{\prime}$.

4. Return $m$.

\subsection{Commitment Schemes}

Recall we have three types of commitment schemes; statistically binding, statistically hiding and computationally binding/hiding, to commit to a value $m \in$ $\{0,1\}^{t}$. (Note that the elliptic curve $E$ is of order $q \approx 2^{2 t}$ and so we can view $m$ as a number in $\mathbb{Z}_{q}$ if desired.)

\section{A Statistically Binding Commitment $: \operatorname{com}_{b}(m)$}

We define the statistically binding commitment scheme as in Figure 2. The random oracle model based scheme is statistically binding, since to break the binding 
property we need to find collisions in the hash function $H$. Since $H$ is modelled as a random oracle, the probability of any adversary finding a collision given a polynomial number of points of $H$ is negligible, even if it is computationally unbounded. The scheme is also computationally hiding by the fact that $H$ is modelled as a random oracle (in fact, it's even statistically hiding if the adversary is limited to a polynomial number of points of $H$ ). The non-ROM scheme is statistically binding because $P$ and $c_{1}$ fully determine $r$, which together with $Q$ and $c_{2}$ in turn fully determine $m$. The fact that it is computationally hiding follows directly from the DDH assumption over the elliptic curve used.

Figure 2 ROM and non-ROM statistically binding commitment schemes

\section{ROM Version}

$H$ is a hash function modeled as a random oracle.

Commitment $\operatorname{com}_{b}(m)$

1. $r \leftarrow\{0,1\}^{t}$.

2. $c \leftarrow H(m \| r)$.

3. Return $c$.

\section{Decommitment}

1. Reveal $m$ and $r$.

2. Check if $c=H(m \| r)$.

3. Return $m$.

\section{Non-ROM Version}

$P$ and $Q$ are elements on an elliptic curve, as described above.

Commitment $\operatorname{com}_{b}(m)$

1. $r \leftarrow \mathbb{Z}_{q}$.

2. $c_{1} \leftarrow[r] P$.

3. $c_{2} \leftarrow[r][m] Q$.

4. Return $\left(c_{1}, c_{2}\right)$.

\section{Decommitment}

1. Reveal $m$ and $r$.

2. Check if $c_{1}=[r] P$.

3. Check if $c_{2}=[r][m] Q$.

4. Return $m$.

The Statistically Hiding Commitment : $\operatorname{com}_{h}(m)$

For the statistically hiding commitment scheme we use the Pederson commitment [18]:

$$
\operatorname{com}_{h}(m) \leftarrow[r] P+[m] Q
$$

where $r$ is a random number of size $q$ and we treat $m$ as an integer modulo $q$. Note that $0 \leq m<2^{t}<q<2^{2 t}$. Decommitment is performed by revealing $r$ and $m$, and then verifying the commitment is valid. This is actually a perfectly hiding commitment (since given $\operatorname{com}_{h}(m)$ there exists, for any possible value of $m^{\prime}$, a corresponding value $r^{\prime}$ for which $\left.\operatorname{com}_{h}(m)=\left[r^{\prime}\right] P+\left[m^{\prime}\right] Q\right)$ and so in particular the commitment is also statistically hiding. That the commitment is computationally binding follows from the fact that any adversary who can break the binding property can determine the discrete logarithm of $Q$ with respect to $P$.

\section{A Computational Commitment Scheme $: \operatorname{com}_{c}(m)$}

We use the ROM version of the statistically binding commitment scheme in 
Figure 2 for both the ROM and non-ROM commitments here. This is clearly suitable in the ROM. Regarding the non-ROM case, this scheme is computationally binding on the assumption that $H$ is collision-resistant. Furthermore, it is computationally hiding when $H(m \| r)$ is modelled as a PRF with key $r$ and message $m$. We remark that when $m$ is large, this latter assumption clearly does not hold for typical hash functions based on the Merkle-Damgård paradigm (where given $H(k \| m)$ one can easily compute $H\left(k\|m\| m^{\prime}\right)$ for some $\left.m^{\prime}\right)$. However, it is reasonable when $m$ fits into a single iteration of the underlying compression function (as is the case here where $m \in\{0,1\}^{t}$ and $t$ is a computational security parameter which we set to the value $t=128$.).

\subsection{Oblivious Transfer}

Recall in our main protocol we need to perform $w=\max \left(4 n, 8 s_{2}\right)$ 1-out-of2 oblivious transfers in Stage 2. We batch these up so as to perform all the OT's in a single batch. The OT's need to be performed in a manner which has a simulation based proof of security against malicious adversaries, hence the simple protocols of $[17,1,12]$ are not suitable for our purposes (the simulation based proof is needed in order to be able to use a composition of the OT protocol in our protocol, see [6]). We therefore use a modification of the batched version of the protocol of Hazay and Lindell [10], which we now describe in the elliptic curve setting. (We note that this protocol has a simulation based proof of security in the standard model, without any usage of a random oracle.)

We assume that $P_{1}$ 's input is two vectors of values

$$
\left[x_{1}^{0}, \ldots, x_{w}^{0}\right] \text { and }\left[x_{1}^{1}, \ldots, x_{w}^{1}\right]
$$

where $\left|x_{j}^{0}\right|=\left|x_{j}^{1}\right|$. Party $P_{2}$ has as input the bits $i_{1}, \ldots, i_{w}$ and wishes to obtain the vector $\left[x_{1}^{i_{1}}, \ldots, x_{w}^{i_{w}}\right]$.

We assume two zero-knowledge proofs-of-knowledge protocols which we shall describe in Appendix A. The first, $D L([x] P ; x)$, proves, in zero-knowledge, knowledge of the discrete logarithm $x$ of $[x] P$; the second, $D H(P,[a] P,[b] P,[a b] P)$, proves that the tuple $P,[a] P,[b] P,[a b] P$ is a Diffie-Hellman tuple.

The protocol follows. The main things to notice are that the zero-knowledge proofs of knowledge are performed only once, regardless of the number of items to be transfered, and that protocol is composed of only two rounds (in addition to the rounds needed by the zero-knowledge proofs).

1. $P_{2}$ chooses $\alpha_{0}, \alpha_{1} \in \mathbb{Z}_{q}$ and computes $Q_{0} \leftarrow\left[\alpha_{0}\right] P$ and $Q_{1} \leftarrow\left[\alpha_{1}\right] P$, it then executes the protocol $D L\left(Q_{0} ; \alpha_{0}\right)$ with party $P_{1}$.

2. For $j=1, \ldots, w$ party $P_{2}$ chooses $r_{j} \in \mathbb{Z}_{q}$ and computes $U_{j} \leftarrow\left[r_{j}\right] P$, $V_{0, j} \leftarrow\left[r_{j}\right] Q_{0}+\left[i_{j}\right] P, V_{1, j} \leftarrow\left[r_{j}\right] Q_{1}+\left[i_{j}\right] P$. These values are then sent to $P_{1}$.

3. $P_{1}$ chooses $\rho_{j} \in \mathbb{Z}_{q}$, for $j=1, \ldots, w$ and sends them to $P_{2}$. 
4. Both parties then locally compute

$$
U \leftarrow \sum_{j=1}^{w}\left[\rho_{j}\right] U_{j}, \quad V \leftarrow \sum_{j=1}^{w}\left[\rho_{j}\right]\left(V_{0, j}-V_{1, j}\right) .
$$

Party $P_{2}$ executes the protocol $D H\left(P, Q_{0}-Q_{1}, U, V\right)$ with party $P_{1}$.

5. For $j=1, \ldots, w P_{1}$ then performs the following steps:

(a) Select $R_{0, j}, R_{1, j} \in\langle P\rangle$ at random.

(b) Select $s_{0, j}, t_{0, j}, s_{1, j}, t_{1, j} \in \mathbb{Z}_{q}$.

(c) Set $e_{0, j} \leftarrow\left(W_{0, j}, Z_{0, j}, y_{0, j}\right)$ where

$$
\begin{aligned}
W_{0, j} & \leftarrow\left[s_{0, j}\right] U+\left[t_{0, j}\right] P, \\
Z_{0, j} & \leftarrow\left[s_{0, j}\right] V_{0}+\left[t_{0, j}\right] Q_{0}+R_{0, j}, \\
y_{0, j} & \leftarrow x_{j}^{0} \oplus \operatorname{KDF}\left(R_{0, j},\left|x_{j}^{0}\right|\right) .
\end{aligned}
$$

(d) Set $e_{1, j} \leftarrow\left(W_{1, j}, Z_{1, j}, y_{1, j}\right)$ where

$$
\begin{aligned}
W_{1, j} & \leftarrow\left[s_{1, j}\right] U+\left[t_{1, j}\right] P, \\
Z_{1, j} & \leftarrow\left[s_{1, j}\right]\left(V_{1}-P\right)+\left[t_{1, j}\right] Q_{1}+R_{1, j}, \\
y_{1, j} & \leftarrow x_{j}^{1} \oplus \operatorname{KDF}\left(R_{1, j},\left|x_{j}^{1}\right|\right) .
\end{aligned}
$$

The values $\left(e_{0, j}, e_{1, j}\right)$ are then sent to $P_{2}$ for each value of $j$.

6. For $j=1, \ldots, w$, party $P_{2}$ then computes

$$
R \leftarrow Z_{i_{j}, j}-\left[\alpha_{i_{j}}\right] W_{i_{j}, j}
$$

and outputs

$$
x_{j}^{i_{j}} \leftarrow y_{i_{j}, j} \oplus \operatorname{KDF}\left(R,\left|x_{j}^{i_{j}}\right|\right) .
$$

For each index in the vector of inputs, the protocol requires $P_{1}$ to perform 10 multiplications, and $P_{2}$ to perform 8 multiplications. (This is without considering the zero-knowledge proofs, which are performed once in the protocol.) The security of the above scheme is fully proven in [10], with the only exception that here a KDF is used to derive a random string in order to mask (i.e., encrypt) the $x_{j}^{0}$ and $x_{j}^{1}$ values (in [10] it is assumed that $x_{j}^{0}$ and $x_{j}^{1}$ can be mapped into points in the Diffie-Hellman group). The use of a KDF for this purpose was proven secure in the context of hashed ElGamal in [22], on the assumption that KDF is chosen from a family of hash functions which are entropy smoothing.

\section{$5 \quad$ Timings}

In our implementation we selected $t=128$ as the security parameter. As a result, we chose the KDF to be implemented by SHA-256, and as the elliptic curve $E$ we selected the curve secp256r1 from the SECG standard [20].

We performed a set of experiments which examined the system using a circuit which evaluates the function $x>y$ for inputs $x$ and $y$ of $n=16$ bits in length. 
The standard circuit (using simple 2-to-1 gates) for this problem consists of 61 2-to-1 gates and 93 internal wires. We optimized this circuit by replacing it with a circuit consisting of 48 internal wires and fifteen 3 -to- 1 gates and one 2-to-1 gate. We only looked at the case of $P_{2}$ obtaining the result, the extension to the first party obtaining the result is standard and requires an extension to the circuit to be made, for which similar optimizations can be made.

The size of the modified circuit: Step 0 of the protocol replaces the circuit with a different one which has $\max \left(4 n, 8 s_{2}\right)$ input wires. The statistical security parameter $s_{2}$ therefore affects the size of the circuit, both in terms of the number of wires and the number of gates. When $n<2 s_{2}$, as in our experiments, we have $8 s_{2}$ new input wires. Each original input wire is replaced with the exclusive-or of about $4 s_{2}$ input wires, which can be computed using $4 s_{2}-1$ gates. The circuit therefore grows by about $4 n s_{2}$ gates, which in our case translate to 2560 gates for $s_{2}=40$, and 3840 gates for $s_{2}=60$. We managed to optimize this construction by using a variant of structured Gaussian elimination in order to reuse gates. As a result, for the case of $s_{2}=40$, the augmented circuit produced in Stage 0 has over one thousand gates and over one thousand five hundred internal wires. If $s_{2}$ is increased to 60 then the augmented circuit now has over one thousand five hundred gates and over two thousand internal wires. The exact increase in size depends on the random choices made in Stage 0, but the above values are indicative.

Implementation: The program was implemented in $\mathrm{C}++$ using standard libraries; the elliptic curve routines made use of specially written assembly functions to perform the arithmetic instructions. On the machine that was used for the experiments, and the curve we were using, the software needed 3.9 milliseconds for a basic multiplication, 1.2 milliseconds to multiply the fixed generator, and 5.1 milliseconds in order to compute $(a P+b Q)$ (using a variant of the method described in Algorithm 14.88 of [16]).

The input to the program was a circuit represented by a text file, each line of the text file represented a gate. For example the line

\section{$2110 \quad 16320100$}

represents a 2-to- 1 gate which has input wires numbered 0 and 16 and produces the output wire 32 . The value of the gate is given by the string which follows. The above example implements a two-bit "less than" gate, namely it will output a 1 on wire 32 only if $w_{0}<w_{16}$, i.e. the value of wire 0 is zero and the value of wire 16 is one.

Experiments: We performed a set of experiments with different values of the statistical security parameters $s_{1}$ and $s_{2}$, and using both the ROM and standard model versions of the protocol. The run times, in seconds, are presented in Table 1 , and are reported for each step of the protocol. Timings are performed using the standard Linux system timing facilities, and are as such only indicative. The wall time is measured using the standard time function and the system and user times are measured using the getrusage function. The wall time represents 
the elapsed wall clock time in running the program, the user time represents the amount of time each party actually performed some computation, whereas the syst time represents the time spent by each party in system routines (for example transmitting data, or writing to disk, etc.). All timings were performed on an Intel Core 26420 running at $2.13 \mathrm{GHZ}$ with $4096 \mathrm{~KB}$ of cache and $2 \mathrm{~GB}$ of RAM and are given in seconds.

Basic observations: The computation is not instantaneous but overall the run time is quite reasonable (the overall run time is about 2-3 minutes for a security parameter $\left.s_{1}=160\right)$. The run time is affected, of course, by the fact that 160 copies of the circuit are being used in the computation (compared to a protocol secure against semi-honest adversaries, which uses only a single copy of the circuit), and the fact that each circuit is much larger than its original form (in the experiment more than 1000 gates are added to the circuit in Step 0, where the original circuit consisted of less than 20 gates).

Oblivious transfers: It is a little surprising that Step 2, which includes the oblivious transfers, is not the main bottleneck of the protocol. This is true even though we implemented an OT protocol which is secure against malicious adversaries according to a full simulation definition.

Preprocessing: About half of the run time is consumed by Step 1, where $P_{1}$ prepares the circuits and the commitments. This step can be run offline, before the inputs are known, reducing the online run time by about $50 \%$.

Scaling: Increasing $s_{1}$ by a factor of $c_{1}$ increases by a factor of $c_{1}^{2}$ the number of commitments generated by $P_{1}$ in Step 1 , and increases the number of circuits by $c_{1}$. Increasing $s_{2}$ by a factor of $c_{2}$ increases the size of the modified part of the circuit (which is the bulk of the circuit in our experiments) by a factor of $c_{2}$, and therefore the total size of the circuits is increased by a factor of $c_{1} c_{2}$. In the experiments, we increased both $s_{1}$ and $s_{2}$ by a factor of 1.5 (from 40 to 60 , and from 160 to 240 , respectively). We therefore expected the overhead to increase by a factor of 2.25. The actual measurements showed an increase by a factor slightly larger than 2 .

We did not conduct experiments with circuits of different sizes. When all other parameters are fixed, we expect the run time to be linear in the size of the modified circuit (after the modifications done in Step 0). We can estimate the size of the modified circuit as follows: If $P_{2}$ has $n$ input wires in the original circuit, then the modified circuit is expected to have about $\frac{n}{2} \max \left(4 n, 8 s_{2}\right)$ more gates. (Applying structured Gaussian elimination can enable us to reuse gates and minimize the size of the modified circuit.)

Performance in the ROM and in the standard model: What is interesting about the timings is that there is very little difference between the timings in the ROM and those in the standard model. In Step 1 the ROM version is more efficient simply due to the slightly more efficient encryption scheme used. ${ }^{7}$

\footnotetext{
7 The KDF is invoked in the standard model protocol about twice as many times
} as in the ROM protocol (since the encryption function in the standard model calls 
Given the large number of encryptions needed to produce the garbled circuit this translates into a small advantage for the ROM version compared to the standard-model implementation. For a similar reason one obtains a performance improvement in the ROM in Step 7 in which the circuit is evaluated by $P_{2}$. The decrease in performance of the ROM compared to the standard model in Step 3 we cannot explain, but it is likely to be caused by experimental error.

In viewing the timings it should be born in mind that the main place that the random oracle model is used is in the oblivious transfers in Step 2. At this point we use the ROM to reduce the round complexity of the two required zero-knowledge proofs (see Appendix A for details of this). However, these two proofs are only used once in the whole run of the protocol as we have batched the oblivious transfers, and therefore the run time of Step 2 is about the same in both the ROM and the standard model protocols.

What is surprising about the fact that the standard model is comparable in performance to the ROM is that for simpler cryptographic functionalities, such as encryption or signature schemes, the performance of the best ROM based scheme is often twice as fast as the best known standard model scheme.

\section{Future Work}

An obvious task is to develop the current implementation into a complete system for secure computation. In particular, the system should include a front end that will enable users to provide a high-level specification of the function that they want to compute, and specify the different security parameters that shall be used. A natural approach for this task would be to modify the FairPlay compiler [15] to support our implementation.

The performance of the system is greatly affected by the circuit modification in Step 0 of the protocol, which increases the number of inputs and the size of the circuit. We implemented this step according to the randomized construction in [13]. Another option is to use a linear error-correction code for defining the relation between the original and new input wires of the circuit. (A careful examination of the proof in [13] shows that this is sufficient.) We need an $[N, k, d]$ linear binary code which encodes $k$ bit words into $N$ bit words with a distance of $d=s_{2}$ (say, $d=40$ ). The parameter $k$ corresponds to the number of original input wires of $P_{2}$, while $N$ corresponds to the number of new input wires. The code should satisfy two criteria: (1) the rate $k / N$ should be as high as possible, to keep the number of new input wires close to the number of original input wires, and (2) the block length $k$ should be minimized, to enable the code to be applied (and the rate $k / N$ to be achieved) even if $P_{2}$ 's input is relatively short.

the KDF twice). The increase in the run time of Step 1 when changing the ROM implementation to the standard-model implementation (for $s_{1}=160$ ) is from 60sec to $67 \mathrm{sec}$. We therefore estimate that the circuit construction (Step 1(a)) takes about 7 seconds in the ROM protocol and 14 seconds in the standard model protocol. 
Run Times in the Random Oracle Model

\begin{tabular}{|l|cccccccc|c|}
\hline & \multicolumn{7}{|c|}{ Step } & \\
Time & 1 & 2 & 3 & 4 & 5 & 6 & 7 & 8 & Total \\
\hline \hline$P_{1}, s_{1}=160$, & $s_{2}=$ & 40 \\
\hline Wall & 74 & 20 & 24 & 0 & 7 & 10 & 0 & 0 & 135 \\
User & 60 & 17 & 12 & 0 & 3 & 4 & 0 & 0 & \\
Syst & 16 & 2 & 3 & 0 & 0 & 0 & 0 & 0 & \\
\hline$P_{2}, s_{1}=160, s_{2}=$ & 40 & & \\
\hline Wall & 74 & 20 & 24 & 0 & 8 & 9 & 35 & 1 & 171 \\
User & 0 & 8 & 14 & 0 & 8 & 7 & 29 & 1 & \\
Syst & 0 & 0 & 10 & 0 & 2 & 4 & 8 & 0 & \\
\hline \hline$P_{1}, s_{1}=240$, & $s_{2}=$ & 60 & & \\
\hline Wall & 159 & 34 & 51 & 0 & 19 & 13 & 0 & 0 & 276 \\
User & 123 & 30 & 24 & 0 & 11 & 6 & 0 & 0 & \\
Syst & 35 & 2 & 9 & 0 & 1 & 0 & 0 & 0 & \\
\hline$P_{2}, s_{1}=240$, & $s_{2}=$ & 60 & & \\
\hline Wall & 159 & 34 & 51 & 0 & 19 & 13 & 78 & 3 & 358 \\
\hline User & 0 & 12 & 28 & 0 & 17 & 10 & 61 & 2 & \\
Syst & 0 & 0 & 22 & 0 & 7 & 5 & 18 & 0 & \\
\hline
\end{tabular}

Run Times in the standard Model

\begin{tabular}{|l|cccccccc|c|}
\hline & \multicolumn{7}{|c|}{ Step } & \\
\hline Time & 1 & 2 & 3 & 4 & 5 & 6 & 7 & 8 & Total \\
\hline \hline$P_{1}, s_{1}=160$, & $s_{2}=$ & 40 \\
\hline Wall & 84 & 20 & 24 & 0 & 7 & 7 & 0 & 0 & 142 \\
User & 67 & 18 & 10 & 0 & 5 & 3 & 0 & 0 & \\
Syst & 15 & 0 & 5 & 0 & 0 & 0 & 0 & 0 & \\
\hline$P_{2}, s_{1}=160, s_{2}=$ & $=40$ & \\
\hline Wall & 84 & 20 & 24 & 0 & 7 & 7 & 40 & 2 & 184 \\
User & 0 & 10 & 13 & 0 & 7 & 5 & 32 & 4 & \\
Syst & 0 & 0 & 11 & 0 & 1 & 3 & 8 & 2 & \\
\hline$P_{1}, s_{1}=240, s_{2}=$ & 60 & & \\
\hline Wall & 181 & 35 & 45 & 0 & 18 & 12 & 0 & 0 & 291 \\
User & 145 & 30 & 24 & 0 & 8 & 8 & 0 & 0 & \\
Syst & 35 & 0 & 7 & 0 & 1 & 2 & 0 & 0 & \\
\hline$P_{2}, s_{1}=$ & 240, & $s_{2}=$ & 60 & & \\
\hline Wall & 181 & 35 & 45 & 0 & 18 & 12 & 87 & 5 & 362 \\
User & 0 & 12 & 23 & 0 & 15 & 9 & 70 & 7 & \\
Syst & 0 & 0 & 21 & 0 & 4 & 3 & 20 & 0 & \\
\hline
\end{tabular}

Table 1. Run times of our experiments.

\section{References}

1. B. Aiello, Y. Ishai, and O. Reingold. Priced Oblivious Transfer: How to Sell Digital Goods. In EUROCRYPT 2001, Springer-Verlag (LNCS 2045), 119-135, 2001.

2. G. Aggarwal, N. Mishra, and B. Pinkas. Secure Computation of the k-th Ranked Element. In EUROCRYPT 2004, Springer-Verlag (LNCS 3027), 40-55, 2004.

3. A. Ben-David, N. Nisan and B. Pinkas, FairplayMP - A System for Secure MultiParty Computation, manuscript, 2008.

4. P. Bogetoft, D.L. Christensen, I. Dåmgard, M. Geisler, T. Jakobsen, M. Krøigaard, J.D. Nielsen, J.B. Nielsen, K. Nielsen, J. Pagter, M. Schwartzbach and T. Toft, Multiparty Computation Goes Live, Cryptology ePrint Archive 2008/068, 2008.

5. P. Bogetoft, I. Damgård, T. Jakobsen, K. Nielsen, J. Pagter. and T. Toft. A practical implementation of secure auctions based on multiparty integer computation. In Financial Cryptography and Data Security - FC 2006, Springer-Verlag LNCS 4107, 142-147, 2006.

6. R. Canetti. Security and Composition of Multiparty Cryptographic Protocols. Journal of Cryptology, 13(1):143-202, 2000.

7. D. Chaum and T.P. Pederson. Wallet Databases with Observers. In Advances in Crytology - Crypto '92, Springer-Verlag LNCS 740, 89-105, 1992.

8. O. Goldreich. Foundations of Cryptography: Volume 2-Basic Applications. Cambridge Univ. Press, 2004.

9. O. Goldreich, S. Micali and A. Wigderson. How to Play any Mental Game - A Completeness Theorem for Protocols with Honest Majority. In 19th STOC, pages 218-229, 1987. 
10. C. Hazay and Y. Lindell. Oblivious transfer, polynomial evaluation and set intersection. Manuscript, 2008.

11. S. Jarecki and V. Shmatikov. Efficient two-party secure computation on committed inputs. In Advances in Cryptology - EuroCrypt 2007, LNCS 4515, 97-114, 2007.

12. Y.T. Kalai. Smooth Projective Hashing and Two-Message Oblivious Transfer. In EUROCRYPT 2005, Springer-Verlag (LNCS 3494), pages 78-95, 2005.

13. Y. Lindell and B. Pinkas. An efficient protocol for secure two-party computation in the presence of malicious adversaries. In Advances in Cryptology-EUROCRYPT 2007, Springer-Verlag LNCS 4515, 52-78, 2007.

14. D. Malkhi and M.K. Franklin. Efficiency tradeoffs for malicious two-party computation. In Public Key Cryptography - PKC 2006, LNCS 3958, 458-473, 2006.

15. D. Malkhi, N. Nisan, B. Pinkas and Y. Sella. Fairplay - a secure two-party computation system. In Proc. of 13th USENIX Security Symposium, 2004.

16. A.J. Menezes, P.C. van Oorschot and S.A. Vanstone, Handbook of Applied Cryptography, CRC Press, 1996

17. M. Naor and B. Pinkas. Efficient oblivious transfer protocols. In 12th SODA, 448-457, 2001.

18. T.P. Pederson. Non-interactive and information-theoretic secure verifiable secret sharing. In Advanced in Cryptology - CRYPTO '91, LNCS 576, 129-140, 1992.

19. Standards for Efficient Cryptography, SEC 1: Elliptic Curve Cryptography. Available from http://www.secg.org/download/aid-385/sec1_final.pdf

20. SECG. Standards for Efficient Cryptography, SEC 2: Recommended elliptic curve domain parameters. Available from http://www.secg.org.

21. C.P. Schnorr. Efficient identification and signatures for smart cards. In Advances in Cryptology - Crypto '89, Springer-Verlag LNCS 435, 239-252, 1990.

22. V. Shoup. Sequences of games: A tool for taming complexity in security proofs. Manuscript, 2004.

23. D. Woodruff. Revisiting the Efficiency of Malicious Two-Party Computation. In Eurocrypt '07, Springer-Verlag (LNCS 4515), pages 79-96, 2007.

24. A. Yao. How to generate and exchange secrets. In 27th FOCS, 162-167, 1986.

\section{A Zero Knowledge Proofs}

We now describe the zero-knowledge proof-of-knowledge protocols required in the OT protocol. In the ROM we use the standard Fiat-Shamir transform of an interactive honest-verifier $\Sigma$-protocol into a non-interactive protocol via hashing the commitment with the random oracle so as to produce the random challenge.

In the standard model we need to cope with non-honest verifiers by getting the verifier to commit to his challenge before the prover's commitment is issued. We use a highly-efficient transformation described in [10] to transform an honest-verifier Sigma protocol to a protocol that is a zero-knowledge proof of knowledge (the transformation is proven secure under the assumption that the discrete logarithm problem is hard and hence is highly suitable for proofs of Diffie-Hellman type statements).

\section{A.1 $D L(Q ; x)$}

We assume a prover Pro who knows $x$ and a verifier $\operatorname{Ver}$ who only knows $Q$ 
and $P$. The two protocols, one in the ROM and one in the standard model, are presented in Fig. 3. They are based on the HVZK proof of Schnorr [21].

$\overline{\text { Figure } 3 \mathrm{ROM} \text { and non-ROM zero-knowledge proof of knowledge of discrete }}$ logarithms

\section{ROM Version}

- Pro computes $k \leftarrow \mathbb{Z}_{q}, R \leftarrow[k] P, s \leftarrow H(R), z \leftarrow x s+k$. It sends $R$ and $z$ to Ver.

- Ver computes $s \leftarrow H(R)$. and accepts if $[z] P=[s] Q+R$.

\section{Non-ROM Version}

- Pro computes $a \leftarrow \mathbb{Z}_{q}, A \leftarrow[a] P$. It sends $A$ to Ver.

- Ver computes $s, t \leftarrow \mathbb{Z}_{q}, C \leftarrow[s] P+[t] A$. and sends $C$ to Pro.

- Pro computes $k \leftarrow \mathbb{Z}_{q}, R \leftarrow[k] P$. and sends $R$ to Ver.

- Ver sends $s, t$ to Pro.

- Pro checks whether $C=[s] P+[t] A$. and sends $z \leftarrow x s+k$ and $a$ to Ver.

- Ver accepts if $[z] P=[s] Q+R$ and $A=[a] P$.

\section{A.2 $D D H(P,[a] P,[b] P,[a b] P)$}

We assume a prover Pro who knows $b$ and a verifier $V e r$ who only knows the four protocol inputs $P, Q=[a] P, U=[b] P$ and $V=[b] Q$. The two variants of the protocol are given in Fig. 4, both are based on the HVZK protocol from [7].

$\overline{\text { Figure } 4 \text { ROM and non-ROM zero-knowledge proof of knowledge of DDH tuple }}$

\section{ROM Version}

- Pro computes $r \leftarrow \mathbb{Z}_{q}, A \leftarrow[r] P, B \leftarrow[r] Q, s \leftarrow H(A \| B), z \leftarrow b s+r$. and sends $A, B$ and $z$ to Ver.

- Ver computes $s \leftarrow H(A \| B)$ and accepts if $[z] P=[s] U+A$ and $[z] Q=[s] V+B$.

\section{Non-ROM Version}

- Pro computes $w \leftarrow \mathbb{Z}_{q}, W \leftarrow[w] P$ and sends $V$ to Ver.

- Ver computes $s, t \leftarrow \mathbb{Z}_{q}, C \leftarrow[s] P+[t] A$ and sends $C$ to Pro.

- Pro computes $r \leftarrow \mathbb{Z}_{q}, A \leftarrow[r] P, B \leftarrow[r] Q$ and sends $A$ and $B$ to Ver.

- Ver sends $s, t$ to Pro.

- Pro checks whether $C=[s] P+[t] V$. and sends $z \leftarrow b s+r$ and $w$ to Ver.

- Ver accepts if $[z] P=[s] U+A,[z] Q=[s] V+B$ and $W=[w] P$. 